\title{
Multiplicity of Nontrivial Solutions for a Class of Nonlocal Elliptic Operators Systems of Kirchhoff Type
}

\author{
Yuping $\mathrm{Cao}^{1}$ and Chuanzhi Bai ${ }^{2}$ \\ ${ }^{1}$ Department of Basic Courses, Lianyungang Technical College, Lianyungang, Jiangsu 222000, China \\ ${ }^{2}$ Department of Mathematics, Huaiyin Normal University, Huaian, Jiangsu 223300, China
}

Correspondence should be addressed to Chuanzhi Bai; czbai@hytc.edu.cn

Received 22 May 2014; Revised 2 July 2014; Accepted 2 July 2014; Published 13 July 2014

Academic Editor: Julio D. Rossi

Copyright (c) 2014 Y. Cao and C. Bai. This is an open access article distributed under the Creative Commons Attribution License, which permits unrestricted use, distribution, and reproduction in any medium, provided the original work is properly cited.

We investigate the existence and multiplicity of nontrivial solutions for a Kirchhoff type problem involving the nonlocal integrodifferential operators with homogeneous Dirichlet boundary conditions. The main tool used for obtaining our result is Morse theory.

\section{Introduction}

This paper is concerned with the multiplicity of solutions to the following elliptic systems of Kirchhoff type involving the nonlocal integrodifferential operators:

$$
\begin{aligned}
& -M_{1}\left(\int_{\mathbb{R}^{2 n}}|u(x)-u(y)|^{2} K_{1}(x-y) d x d y\right) \mathscr{L}_{K_{1}} u \\
& =f(x, v) \quad \text { in } \Omega, \\
& -M_{2}\left(\int_{\mathbb{R}^{2 n}}|v(x)-v(y)|^{2} K_{2}(x-y) d x d y\right) \mathscr{L}_{K_{2}} v \\
& =g(x, u) \quad \text { in } \Omega, \\
& u=v=0 \quad \text { in } \mathbb{R}^{n} \backslash \Omega,
\end{aligned}
$$

where $\Omega \subset \mathbb{R}^{n}(n \geq 2)$ is a bounded domain with smooth boundary $\partial \Omega$ and $f, g: \Omega \times \mathbb{R} \rightarrow \mathbb{R}$ are two continuous functions. $M_{i}: \mathbb{R}^{+} \rightarrow \mathbb{R}^{+}(i=1,2)$ are two continuous functions whose properties will be introduced later. $\mathscr{L}_{K_{i}}(i=$ $1,2)$ are the nonlocal operators defined by

$$
\begin{array}{r}
\mathscr{L}_{K_{i}} u(x)=\frac{1}{2} \int_{\mathbb{R}^{n}}(u(x+y)+u(x-y)-2 u(x)) K_{i}(y) d y, \\
x \in \mathbb{R}^{n},
\end{array}
$$

$i=1,2$; here $K_{i}: \mathbb{R}^{n} \backslash\{0\} \rightarrow(0,+\infty)$ is a function such that

$$
m K_{i} \in L^{1}\left(\mathbb{R}^{n}\right), \quad i=1,2 \text {, where } m(x)=\min \left\{|x|^{2}, 1\right\} ;
$$

there exist $\theta_{i}$ and $s_{i} \in(0,1)(i=1,2)$ such that

$$
\begin{gathered}
K_{i}(x) \geq \theta_{i}|x|^{-\left(n+2 s_{i}\right)}, \quad \text { for any } x \in \mathbb{R}^{n} \backslash\{0\} ; \\
K_{i}(x)=K_{i}(-x), \quad \forall x \in \mathbb{R}^{n} \backslash\{0\} .
\end{gathered}
$$


A typical example for $K_{i}$ is given by $K_{i}(x)=|x|^{-\left(n+2 s_{i}\right)}$ $(i=1,2)$. In this case $\mathscr{L}_{K_{i}}$ is the fractional Laplace operator $-(-\Delta)^{s_{i}}$, where $-(-\Delta)^{s_{i}}$ is defined by

$$
\begin{array}{r}
-(-\Delta)^{s_{i}} u(x)=\frac{1}{2} \int_{\mathbb{R}^{n}} \frac{u(x+y)+u(x-y)-2 u(x)}{|y|^{n+2 s_{i}}} d y, \\
x \in \mathbb{R}^{n} ;
\end{array}
$$

here $s_{i} \in(0,1)$ and $n>2 s_{i}(i=1,2)$. The fractional Laplacian $-(-\Delta)^{s_{i}}$ is a classical linear integrodifferential operator of order $2 s_{i}$ which gives the standard Laplacian when $s_{i}=1$ (see $[1])$.

Denote by $X_{i}$ the linear space of Lebesgue measurable functions $u: \mathbb{R}^{n} \rightarrow \mathbb{R}$ such that

$$
\begin{aligned}
& \text { the } \operatorname{map}(x, y) \mapsto(u(x)-u(y))^{2} K_{i}(x-y) \\
& \text { is in } L^{1}(Q, d x d y),
\end{aligned}
$$

where $Q=\left(\mathbb{R}^{n} \times \mathbb{R}^{n}\right) \backslash \mathcal{O}$ and $\mathcal{O}=(\mathscr{C} \Omega) \times(\mathscr{C} \Omega) \subset \mathbb{R}^{n} \times \mathbb{R}^{n}$. The space $X_{i}$ is endowed with the norm

$$
\begin{aligned}
&\|u\|_{X_{i}}=\|u\|_{L^{2}\left(\mathbb{R}^{n}\right)} \\
&+\left(\int_{\mathbb{R}^{n} \times \mathbb{R}^{n}}|u(x)-u(y)|^{2} K_{i}(x-y) d x d y\right)^{1 / 2}, \\
& i=1,2 .
\end{aligned}
$$

The space $Z_{i}$ denotes the closure of $C_{0}^{\infty}(\Omega)$ in $X_{i}$. By Lemmas 6 and 7 in [2], the space $Z_{i}$ is a Hilbert space which can be endowed with the norm defined as

$$
\|u\|_{Z_{i}}=\left(\int_{Q}|u(x)-u(y)|^{2} K_{i}(x-y) d x d y\right)^{1 / 2}, \quad i=1,2 .
$$

Since $u=0$ a.e. in $\mathbb{R}^{n} \backslash \Omega$, we have that the integral in (8) and (9) can be extended to all $\mathbb{R}^{2 n}$.

Let $E=Z_{1} \times Z_{2}$ be the Cartesian product of two Hilbert spaces, which is a reflexive Banach space endowed with the norm

$$
\|(u, v)\|=\|u\|_{Z_{1}}+\|v\|_{Z_{2}} .
$$

Denote by $0<\lambda_{1}<\lambda_{2} \leq \cdots \leq \lambda_{k} \leq \cdots$ the eigenvalues of the following nonlocal operator eigenvalue problem:

$$
\begin{gathered}
-\mathscr{L}_{K_{1}} u=\lambda u \quad \text { in } \Omega \\
u=0 \quad \text { in } \mathbb{R}^{n} \backslash \Omega .
\end{gathered}
$$

Similarly, denote by $0<\mu_{1}<\mu_{2} \leq \cdots \leq \mu_{k} \leq \cdots$ the eigenvalues of the following nonlocal operator eigenvalue problem:

$$
\begin{gathered}
-\mathscr{L}_{K_{2}} v=\mu v \text { in } \Omega, \\
v=0 \quad \text { in } \mathbb{R}^{n} \backslash \Omega .
\end{gathered}
$$

We say that $(u, v) \in E$ is a weak solution of system (1) if, for every $(\phi, \psi) \in E$, one has

$$
\begin{gathered}
M_{1}\left(\|u\|_{Z_{1}}^{2}\right) \int_{\mathbb{R}^{2 n}}(u(x)-u(y))(\phi(x)-\phi(y)) \\
\times K_{1}(x-y) d x d y+M_{2}\left(\|v\|_{Z_{2}}^{2}\right) \\
\times \int_{\mathbb{R}^{2 n}}(v(x)-v(y))(\psi(x)-\psi(y)) K_{2}(x-y) d x d y \\
-\int_{\Omega} f(x, v) \phi(x) d x-\int_{\Omega} g(x, u) \psi(x) d x=0 .
\end{gathered}
$$

The fractional Laplacian and nonlocal operators of elliptic type arise in both pure mathematical research and concrete applications, since these operators occur in a quite natural way in many different contexts. For an elementary introduction to this topic, see [2] and the references therein. Recently, some elliptic boundary problems driven by the nonlocal integrodifferential operator $\mathscr{L}_{K}$ have been studied in the works [3-8].

Recently, problems involving Kirchhoff type operators have been studied in many papers; we refer to [9-13] in which the authors have used the variational method and topological method to get the existence of solutions.

In this paper, motivated by the above mentioned works, we will use Morse theory to investigate the multiplicity of solutions of problem (1). To the best of our knowledge, there is no effort being made in the literature to study the existence of solutions for problem (1). This paper will make some contribution to this research field.

In order to establish solutions for problem (1), we make the following assumptions.

(H1) $M_{i}: \mathbb{R}^{+} \rightarrow \mathbb{R}^{+}(i=1,2)$ are two continuous functions, and there exist constants $m_{1}, m_{2}, M_{1}, M_{2}>$ 0 such that

$$
m_{i} \leq M_{i}(t) \leq M_{i}, \quad i=1,2, \forall t \geq 0 .
$$

(H2) $f(x, v)$ and $g(x, u)$ are two continuous functions with the subcritical growth; that is, there exist some positive constants $C_{1}, C_{2}$ such that

$$
\begin{array}{r}
|f(x, v)| \leq C_{1}\left(1+|v|^{p-1}\right), \\
|g(x, u)| \leq C_{2}\left(1+|u|^{q-1}\right), \\
\forall x \in \Omega, \quad u, v \in \mathbb{R}
\end{array}
$$

hold, where $1<p<2_{s_{1}}^{*}=2 n /\left(n-2 s_{1}\right), 1<q<2_{s_{2}}^{*}=$ $2 n /\left(n-s_{2}\right)$. 
(H3) There exists $r>0, \bar{\lambda} \in\left(\lambda_{1}, \lambda_{2}\right)$ and $\bar{\mu} \in\left(\mu_{1}, \mu_{2}\right)$ such that $M_{1} \lambda_{1}<m_{1} \bar{\lambda}, M_{2} \mu_{1}<m_{2} \bar{\mu}$, and $|u|,|v| \leq r$ implies

$$
\begin{array}{r}
\frac{1}{2} M_{2} \mu_{1} v^{2} \leq F(x, v) \leq \frac{1}{2} m_{2} \bar{\mu} v^{2}, \\
\frac{1}{2} M_{1} \lambda_{1} u^{2} \leq G(x, u) \leq \frac{1}{2} m_{1} \bar{\lambda} u^{2}, \\
\text { a.e. } x \in \Omega .
\end{array}
$$

(H4) $\lim _{|v| \rightarrow \infty}\left(F(x, v) / v^{2}\right)<(1 / 2) m_{2} \mu_{1}, \quad \lim _{|u| \rightarrow \infty}$ $\left(G(x, u) / u^{2}\right)<(1 / 2) m_{1} \lambda_{1}$, uniformly for all a.e. $x \in \bar{\Omega}$.

The main result of this paper is as follows.

Theorem 1. If(H1)-(H4) hold, then the problem (1) has at least two nontrivial weak solutions in $E$.

\section{Preliminaries}

For each $(u, v) \in E$, we define the functional $\mathscr{J}: E \rightarrow \mathbb{R}$ as follows:

$$
\begin{aligned}
\mathscr{J}(u, v)= & \frac{1}{2} \widehat{M_{1}}\left(\|u\|_{Z_{1}}^{2}\right)+\frac{1}{2} \widehat{M_{2}}\left(\|v\|_{Z_{2}}^{2}\right) \\
& -\int_{\Omega} F(x, v) d x-\int_{\Omega} G(x, u) d x,
\end{aligned}
$$

where

$$
\begin{gathered}
\widehat{M}_{i}(t)=\int_{0}^{t} M_{i}(\tau) d \tau, \quad i=1,2, \quad t \geq 0, \\
F(x, v)=\int_{0}^{v} f(x, s) d s, \quad G(x, u)=\int_{0}^{u} g(x, s) d s .
\end{gathered}
$$

It is easy to check that $(u, v)$ is a weak solution of problem (1) which is equivalent to being a critical point of the functional $\mathscr{g}$.

First let us recall the definition of the local linking which plays an important role in our paper.

Definition 2. Let $X$ be a Banach space with a direct sum decomposition $X=X^{1} \oplus X^{2}$. The functional $f \in C^{1}(X, \mathbb{R})$ has a local linking at 0 with respect to $\left(X^{1}, X^{2}\right)$ if there is $r>0$ such that

$$
\begin{aligned}
& f(u) \geq 0, \quad \forall u \in X^{1} \text { with }\|u\| \leq r \\
& f(u) \leq 0, \quad \forall u \in X^{2} \text { with }\|u\| \leq r .
\end{aligned}
$$

Lemma 3. Assume that (H1) and (H4) hold; then the functional $\mathscr{F}$ is coercive in $E$; that is, $\mathscr{F}(u, v) \rightarrow+\infty$ as $\|(u, v)\| \rightarrow$ $\infty$.
Proof. From (H4) and the continuity of the potentials $F$ and $G$ we have that, for some $\epsilon>0$, there exists a positive constant $C_{3}$ such that

$$
\begin{array}{r}
F(x, t) \leq \frac{m_{2}}{2}\left(\mu_{1}-\epsilon\right)|t|^{2}+C_{3}, \\
G(x, t) \leq \frac{m_{1}}{2}\left(\lambda_{1}-\epsilon\right)|t|^{2}+C_{3}, \\
\forall t \in \mathbb{R}, \quad \text { a.e. } x \in \Omega .
\end{array}
$$

Thus, by the Sobolev inequality [1] and (H1), for $(u, v) \in E$, we obtain

$$
\begin{aligned}
\mathscr{J}(u, v) \geq & \frac{m_{1}}{2}\|u\|_{Z_{1}}^{2}+\frac{m_{2}}{2}\|v\|_{Z_{2}}^{2}-\frac{m_{1}\left(\lambda_{1}-\epsilon\right)}{2} \int_{\Omega} u^{2} d x \\
& -\frac{m_{2}\left(\mu_{1}-\epsilon\right)}{2} \int_{\Omega} v^{2} d x-2 C_{3}|\Omega| \\
\geq & \frac{m_{1}}{2}\left(1-\frac{\lambda_{1}-\epsilon}{\lambda_{1}}\right)\|u\|_{Z_{1}}^{2}+\frac{m_{2}}{2}\left(1-\frac{\mu_{1}-\epsilon}{\mu_{1}}\right)\|v\|_{Z_{2}}^{2} \\
& -2 C_{3}|\Omega| \longrightarrow+\infty
\end{aligned}
$$

as $\|(u, v)\| \rightarrow \infty$. Hence, we have that $\mathscr{F}$ is coercive in $E$.

Lemma 4. If (H1), (H2), and (H4) hold, then $\mathcal{F}$ satisfies the (P.S.) condition.

Proof. Let $\left\{z_{n}=\left(u_{n}, v_{n}\right)\right\}$ be a (PS) sequence of $\mathscr{F}$; then $\left\{\left(u_{n}, v_{n}\right)\right\}$ must be bounded by Lemma 3. Passing to a subsequence if necessary, there exists $z=(u, v) \in E$ such that $\left(u_{n}, v_{n}\right) \rightarrow(u, v)$ weakly in $E$. Thus, there exists a strictly decreasing subsequence $\epsilon_{n}, \lim _{n \rightarrow \infty} \epsilon_{n}=0$, such that

$$
\left|\mathcal{F}^{\prime}\left(u_{n}, v_{n}\right)\left(u_{n}-u, 0\right)\right| \leq \epsilon_{n}\left\|u_{n}-u, 0\right\| .
$$

In particular,

$$
\begin{aligned}
& \mid M_{1}\left(\left\|u_{n}\right\|_{Z_{1}}^{2}\right) \\
& \quad \times \int_{\mathbb{R}^{2 n}}\left(u_{n}(x)-u_{n}(y)\right) \\
& \quad \times\left(\left(u_{n}-u\right)(x)-\left(u_{n}-u\right)(y)\right) K_{1}(x-y) d x d y \\
& \quad-\int_{\Omega} f\left(x, v_{n}\right)\left(u_{n}-u\right) d x \mid \leq \epsilon_{n}\left\|\left(u_{n}-u, 0\right)\right\| .
\end{aligned}
$$

Since the potential $F$ satisfies (H2) and by remark (3.2.24) in [14] we have

$$
\int_{\Omega} f\left(x, v_{n}\right)\left(u_{n}-u\right) d x \longrightarrow 0 .
$$


Combining (23) with (24), we obtain

$$
\begin{aligned}
m_{1} \mid \int_{\mathbb{R}^{2 n}} & \left(u_{n}(x)-u_{n}(y)\right)\left(\left(u_{n}-u\right)(x)-\left(u_{n}-u\right)(y)\right) \\
\times & K_{1}(x-y) d x d y \mid \\
\leq \mid M_{1}\left(\left\|u_{n}\right\|_{Z_{1}}^{2}\right) \int_{\mathbb{R}^{2 n}} & \left(u_{n}(x)-u_{n}(y)\right) \\
& \times\left(\left(u_{n}-u\right)(x)-\left(u_{n}-u\right)(y)\right) \\
\times & K_{1}(x-y) d x d y \mid
\end{aligned}
$$$$
\longrightarrow 0 \text {. }
$$

On the other hand, we have

$$
\begin{aligned}
\lim _{n \rightarrow \infty} \int_{\mathbb{R}^{2 n}}( & (x(x)-u(y))\left(\left(u_{n}-u\right)(x)-\left(u_{n}-u\right)(y)\right) \\
& \times K_{1}(x-y) d x d y=0 .
\end{aligned}
$$

Adding (25) to (26), we conclude that

$$
\begin{aligned}
0=\lim _{n \rightarrow \infty}[ & \int_{\mathbb{R}^{2 n}}\left(u_{n}(x)-u_{n}(y)\right)^{2} K_{1}(x-y) d x d y \\
& \left.-\int_{\mathbb{R}^{2 n}}(u(x)-u(y))^{2} K_{1}(x-y) d x d y\right],
\end{aligned}
$$

which implies $\left\|u_{n}\right\|_{Z_{1}}^{2} \rightarrow\|u\|_{Z_{1}}^{2}$. So, $\left\|u_{n}\right\|_{Z_{1}} \rightarrow\|u\|_{Z_{1}}$.

Similarly, we can obtain that $\left\|v_{n}\right\|_{Z_{2}} \rightarrow\|v\|_{Z_{2}}$. The uniform convexity of $E$ yields that $\left\{z_{n}\right\}$ converges strongly to $z$ in $E$.

Thanks to the fact that $L^{2_{s_{1}}^{*}}(\Omega) \hookrightarrow L^{p_{1}}(\Omega)\left(2<p_{1}<2_{s_{1}}^{*}\right)$ continuously, we get by Lemma 6 in [2] and (4) that

$$
\begin{aligned}
\|u\|_{L^{p_{1}}(\Omega)} \leq & |\Omega|^{\left(2_{s_{1}}^{*}-p_{1}\right) /\left(p_{1} 2_{s_{1}}^{*}\right)}\|u\|_{L^{2 *} s_{1}(\Omega)} \\
\leq & \left.|\Omega|^{\left(2 s_{1}\right.}-p_{1}\right) /\left(p_{1} 2_{s_{1}}^{*}\right) \\
& \times \sqrt{c_{1}}\left(\int_{\mathbb{R}^{2 n}} \frac{|u(x)-u(y)|^{2}}{|x-y|^{n+2 s_{1}}} d x d y\right)^{1 / 2} \\
\leq & C_{4}\|u\|_{Z_{1}},
\end{aligned}
$$

where $C_{4}=|\Omega|^{\left(2_{s_{1}}^{*}-p_{1}\right) /\left(p_{1} 2_{s_{1}}^{*}\right)} \sqrt{c_{1} / \theta_{1}}$. Similarly, for $2<q_{1}<$ $2_{s_{2}}^{*}$, there exists a constant $C_{5}>0$ such that

$$
\|v\|_{L^{q_{1}}(\Omega)} \leq C_{5}\|v\|_{Z_{2}} .
$$

In the following, set $U=\operatorname{span}\left\{\varphi_{1}\right\} \times \operatorname{span}\left\{\psi_{1}\right\}:=\left\langle\varphi_{1}\right\rangle \times$ $\left\langle\psi_{1}\right\rangle$, where $\varphi_{1}>0$ with $\left\|\varphi_{1}\right\|_{Z_{1}}=1$ is the corresponding eigenfunction of $\lambda_{1}$ and $\psi_{1}>0$ with $\left\|\psi_{1}\right\|_{Z_{2}}=1$ is the corresponding eigenfunction of $\mu_{1}$. Eigenvalues $\lambda_{1}$ and $\mu_{1}$ are as in (11) and (12), respectively. Taking

$$
V=\left\{(u, v) \in E: u \in\left\langle\varphi_{1}\right\rangle^{\perp}, v \in\left\langle\psi_{1}\right\rangle^{\perp}\right\}
$$

we can easily know that $V$ is complementary subspace of $U$. Hence we have the following direct sum:

$$
E=U \oplus V
$$

If $(u, v) \in U$, from Proposition 9 in [4], we get

$$
\|u\|_{Z_{1}}^{2}=\lambda_{1} \int_{\Omega}|u(x)|^{2} d x, \quad\|v\|_{Z_{2}}^{2}=\mu_{1} \int_{\Omega}|v(x)|^{2} d x .
$$

Moreover, if $(u, v) \in V$, by Proposition 9 in [4], we have

$$
\|u\|_{Z_{1}}^{2} \geq \lambda_{2} \int_{\Omega}|u(x)|^{2} d x, \quad\|v\|_{Z_{2}}^{2} \geq \mu_{2} \int_{\Omega}|v(x)|^{2} d x .
$$

Lemma 5. Assume that (H1)-(H3) hold. Then the functional $\mathcal{J}$ has a local linking at the origin with respect to $E=U \oplus V$.

Proof. (i) Let $(u, v) \in U$. Since

$$
\|(u, v)\| \longrightarrow 0 \Longrightarrow \int_{\Omega}|u(x)|^{2} d x \longrightarrow 0, \int_{\Omega}|v(x)|^{2} \longrightarrow 0
$$

by (32), we have that, for given $r>0$, there is some $\rho>0$ small enough such that

$$
(u, v) \in U,\|(u, v)\| \leq \rho \Longrightarrow|u(x)| \leq r,|v(x)| \leq r,
$$

$$
\text { a.e. } x \in \Omega \text {. }
$$

Now on $U$, we have by $(\mathrm{H} 1)$ and $(\mathrm{H} 3)$ that, for $(u, v) \in U$ with $\|(u, v)\| \leq \rho$,

$$
\begin{aligned}
\mathscr{J}(u, v)= & \frac{1}{2} \widehat{M_{1}}\left(\|u\|_{Z_{1}}^{2}\right)+\frac{1}{2} \widehat{M_{2}}\left(\|v\|_{Z_{2}}^{2}\right)-\int_{\Omega} F(x, v) d x \\
& -\int_{\Omega} G(x, u) d x \\
\leq & \frac{M_{1}}{2} \lambda_{1} \int_{\Omega}|u|^{2} d x+\frac{M_{2}}{2} \mu_{1} \int_{\Omega}|v|^{2} d x \\
& -\int_{\Omega} F(x, v) d x-\int_{\Omega} G(x, u) d x \\
= & \int_{|u| \leq r}\left(\frac{1}{2} M_{1} \lambda_{1}|u|^{2}-G(x, u)\right) d x \\
& +\int_{|v| \leq r}\left(\frac{1}{2} M_{2} \mu_{1}|v|^{2}-F(x, v)\right) d x \leq 0 .
\end{aligned}
$$


(ii) Let $(u, v) \in V$. By (33), similar to (34) and (35), we obtain by (H1)-(H3) that, for $(u, v) \in V$ with $\|(u, v)\| \leq \rho$,

$$
\begin{aligned}
& \mathscr{J}(u, v)=\frac{1}{2} \widehat{M_{1}}\left(\|u\|_{Z_{1}}^{2}\right)+\frac{1}{2} \widehat{M_{2}}\left(\|v\|_{Z_{2}}^{2}\right)-\frac{1}{2} m_{1} \bar{\lambda} \int_{\Omega} u^{2} d x \\
& -\frac{1}{2} m_{2} \bar{\mu} \int_{\Omega} v^{2} d x \\
& -\int_{\{|v| \leq r\}}\left(F(x, v)-\frac{1}{2} m_{2} \bar{\mu}|v|^{2}\right) d x \\
& -\int_{\{|v|>r\}}\left(F(x, v)-\frac{1}{2} m_{2} \bar{\mu}|v|^{2}\right) d x \\
& -\int_{\{|u| \leq r\}}\left(G(x, u)-\frac{1}{2} m_{1} \bar{\lambda}|u|^{2}\right) d x \\
& -\int_{\{|u|>r\}}\left(G(x, u)-\frac{1}{2} m_{1} \bar{\lambda}|u|^{2}\right) d x \\
& \geq \frac{m_{1}}{2}\left(1-\frac{\bar{\lambda}}{\lambda_{2}}\right)\|u\|_{Z_{1}}^{2}+\frac{m_{2}}{2}\left(1-\frac{\bar{\mu}}{\mu_{2}}\right)\|v\|_{Z_{2}}^{2} \\
& -\int_{\{|v|>r\}}\left(F(x, v)-\frac{1}{2} m_{2} \bar{\mu}|v|^{2}\right) d x \\
& -\int_{\{|u|>r\}}\left(G(x, u)-\frac{1}{2} m_{1} \bar{\lambda}|u|^{2}\right) d x \\
& \geq \frac{m_{1}}{2}\left(1-\frac{\bar{\lambda}}{\lambda_{2}}\right)\|u\|_{Z_{1}}^{2}+\frac{m_{2}}{2}\left(1-\frac{\bar{\mu}}{\mu_{2}}\right)\|v\|_{Z_{2}}^{2} \\
& -C_{6} \int_{\{|v|>r\}}|v|^{p_{2}} d x-C_{7} \int_{\{|u|>r\}}|u|^{q_{2}} d x \\
& \geq \frac{m_{1}}{2}\left(1-\frac{\bar{\lambda}}{\lambda_{2}}\right)\|u\|_{Z_{1}}^{2}+\frac{m_{2}}{2}\left(1-\frac{\bar{\mu}}{\mu_{2}}\right)\|v\|_{Z_{2}}^{2} \\
& -C_{8}\|u\|_{Z_{1}}^{p_{2}}-C_{9}\|v\|_{Z_{2}}^{q_{2}}, \quad(\text { by }(28)-(29)) \text {, }
\end{aligned}
$$

where $C_{i}(i=6, \ldots, 9)$ are positive constants, $2<p_{2}<2_{s_{1}}^{*}$, and $2<q_{2}<2_{s_{2}}^{*}$. Thus, (37) implies that $\mathscr{J}(u, v)>0$ for $0<\|(u, v)\| \leq \rho$ with $\rho>0$ is small enough. The proof is complete.

Let $X$ be a real Banach space and $f \in C^{1}(X, \mathbb{R})$. Suppose $p$ is an isolated critical point of $f$ with $f(p)=c$ and $U$ is a neighborhood of $p$, containing the unique critical point; the group

$$
C_{q}(f, p)=H_{q}\left(f_{c} \cap U, f_{c} \cap U \backslash\{p\}\right), \quad q=0,1,2, \ldots,
$$

is called the qth critical group of $f$ at $p$, where $f_{c}=\{u \in X$ : $f(u) \leq c\}$ and $H_{q}(\cdot, \cdot)$ is the $q$ th singular relative homology group with integer coefficients.

Lemma 6 (see $[15]$ ). Let $E$ be a Banach space and $f: E \rightarrow \mathbb{R}$ $a C^{1}$-functional satisfying the (P.S) condition. Assume that $f$ has a local linking to the decomposition $E=U \oplus V$ near the origin, where $\operatorname{dim} U=m<\infty$. If $0 \in E$ is the unique critical point of $f$ in $B_{\rho}$, then

$$
C_{m}(f, 0)=H_{m}\left(f_{c} \cap B_{\rho}, f_{c} \cap B_{\rho} \backslash\{0\}\right) \neq 0 .
$$

\section{The Proof of Theorem 1}

We say that $u$ is a homological nontrivial critical point of $f$ if at least one of its critical groups is nontrivial. By [16], we have the following abstract critical point theorem.

Lemma 7 (see [16]). Let $X$ be a real Banach space and let $\Phi \in C^{1}(X, \mathbb{R})$ satisfy the (P.S) condition and be bounded from below. If $\Phi$ has a critical point that is homologically nontrivial and is not the minimizer of $\Phi$, then $\Phi$ has at least three critical points.

From the proof of Lemma 3, we can conclude that $(0,0) \epsilon$ $E$ is the unique critical point of our $\mathscr{J}$ in a ball that is small enough. Since $\operatorname{dim} U=\operatorname{dim}\left\langle\varphi_{1}\right\rangle \times\left\langle\psi_{1}\right\rangle=2<\infty$, by Lemmas 5 and 6 , we have the following lemma.

Lemma 8. Let (H1)-(H3) hold. Then $(0,0)$ is a critical point of $\mathscr{J}$ and $C_{2}(\mathscr{F},(0,0)) \neq 0$.

Proof of Theorem 1. By Lemmas 3 and $4, \mathscr{J}$ is coercive and satisfies the (P.S) condition. Hence $\mathscr{J}$ is bounded below. By Lemma $8,(0,0) \in E$ is homologically nontrivial critical point of $\mathscr{J}$ but not a minimizer. Then the conclusion follows from Lemma 7.

\section{Conflict of Interests}

The authors declare that there is no conflict of interests regarding the publication of this paper.

\section{Acknowledgments}

The authors thank the referees for their valuable and helpful suggestions and comments that improved the paper. This work is supported by the Natural Science Foundation of Jiangsu Province (BK2011407) and the Natural Science Foundation of China (11271364 and 10771212).

\section{References}

[1] E. Di Nezza, G. Palatucci, and E. Valdinoci, "Hitchhiker's guide to the fractional Sobolev spaces," Bulletin des Sciences Mathématiques, vol. 136, no. 5, pp. 521-573, 2012.

[2] R. Servadei and E. Valdinoci, "Mountain pass solutions for nonlocal elliptic operators," Journal of Mathematical Analysis and Applications, vol. 389, no. 2, pp. 887-898, 2012.

[3] L. A. Caffarelli, S. Salsa, and L. Silvestre, "Regularity estimates for the solution and the free boundary of the obstacle problem for the fractional Laplacian," Inventiones Mathematicae, vol. 171, no. 2, pp. 425-461, 2008.

[4] R. Servadei and E. Valdinoci, "Variational methods for nonlocal operators of elliptic type," Discrete and Continuous Dynamical Systems, vol. 33, no. 5, pp. 2105-2137, 2013. 
[5] J. Tan, "The Brezis-Nirenberg type problem involving the square root of the Laplacian," Calculus of Variations and Partial Differential Equations, vol. 36, pp. 21-41, 2011.

[6] B. Barrios, E. Colorado, A. de Pablo, and U. Sánchez, “On some critical problems for the fractional Laplacian operator," Journal of Differential Equations, vol. 252, no. 11, pp. 6133-6162, 2012.

[7] C. Bai, "Existence results for non-local operators of elliptic type," Nonlinear Analysis: Theory, Methods \& Applications, vol. 83, pp. 82-90, 2013.

[8] S. Dipierro and A. Pinamonti, "A geometric inequality and a symmetry result for elliptic systems involving the fractional Laplacian," Journal of Differential Equations, vol. 255, no. 1, pp. 85-119, 2013.

[9] C. Chen, Y. Kuo, and T. Wu, "The Nehari manifold for a Kirchhoff type problem involving sign-changing weight functions," Journal of Differential Equations, vol. 250, no. 4, pp. 1876-1908, 2011.

[10] B. Cheng, "New existence and multiplicity of nontrivial solutions for nonlocal elliptic Kirchhoff type problems," Journal of Mathematical Analysis and Applications, vol. 394, no. 2, pp. 488495, 2012.

[11] D. Liu and P. Zhao, "Multiple nontrivial solutions to a $p$ Kirchhoff equation," Nonlinear Analysis: Theory, Methods \& Applications, vol. 75, no. 13, pp. 5032-5038, 2012.

[12] A. Fiscella and E. Valdinoci, "A critical Kirchhoff type problem involving a nonlocal operator," Nonlinear Analysis: Theory, Methods \& Applications, vol. 94, pp. 156-170, 2014.

[13] C. Chen and Q. Zhu, "Existence of positive solutions to p-Kirchhoff-type problem without compactness conditions," Applied Mathematics Letters, vol. 28, pp. 82-87, 2014.

[14] A. Djellit and S. Tas, "Quasilinear elliptic systems with critical Sobolev exponents in $R^{N}$," Nonlinear Analysis: Theory, Methods \& Applications, vol. 66, no. 7, pp. 1485-1497, 2007.

[15] J. Q. Liu, “The Morse index of a saddle point," Systems Science and Mathematical Sciences, vol. 2, no. 1, pp. 32-39, 1989.

[16] J. Liu and J. Su, "Remarks on multiple nontrivial solutions for quasi-linear resonant problems," Journal of Mathematical Analysis and Applications, vol. 258, no. 1, pp. 209-222, 2001. 


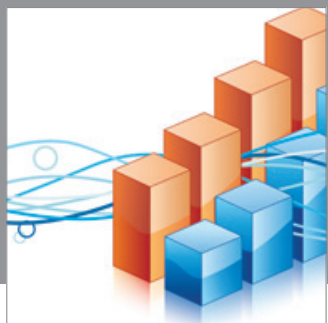

Advances in

Operations Research

mansans

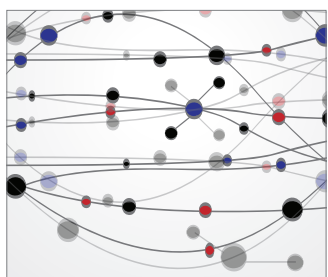

The Scientific World Journal
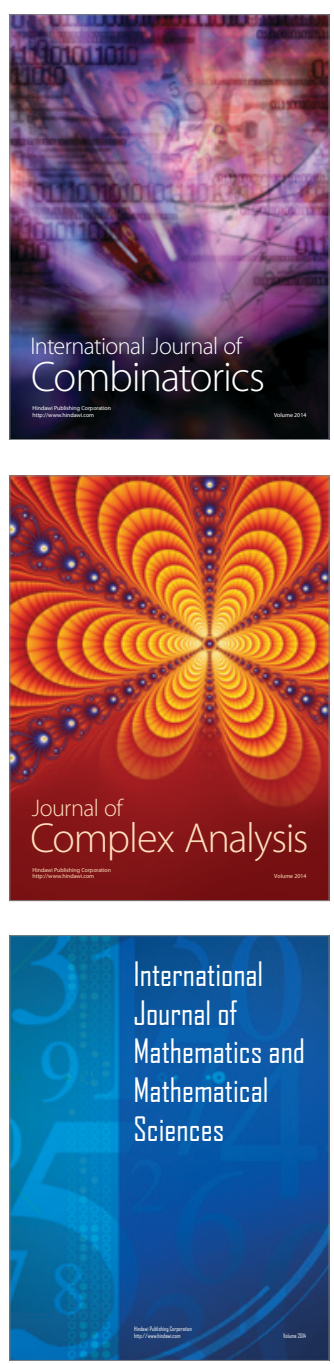
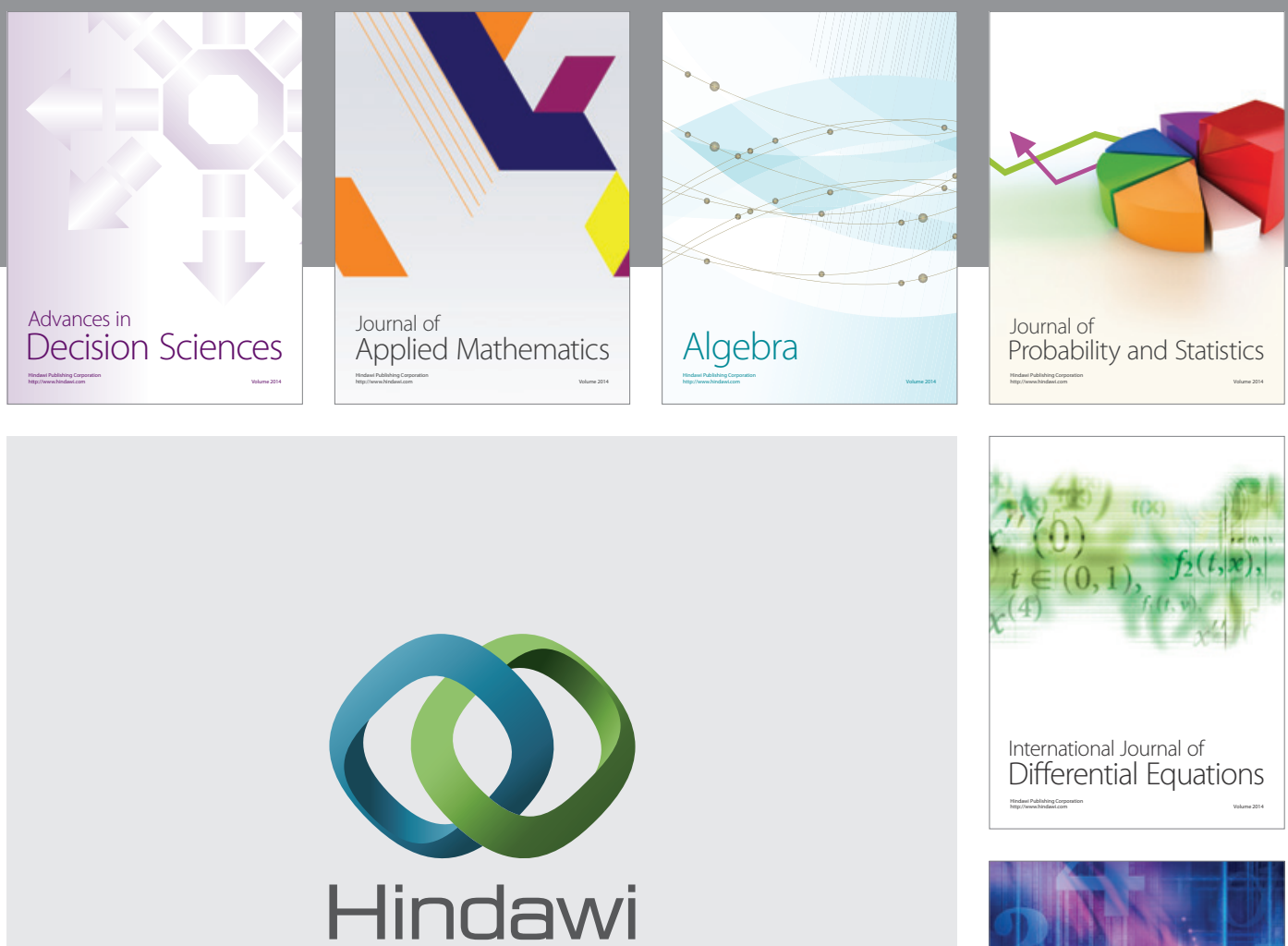

Submit your manuscripts at http://www.hindawi.com
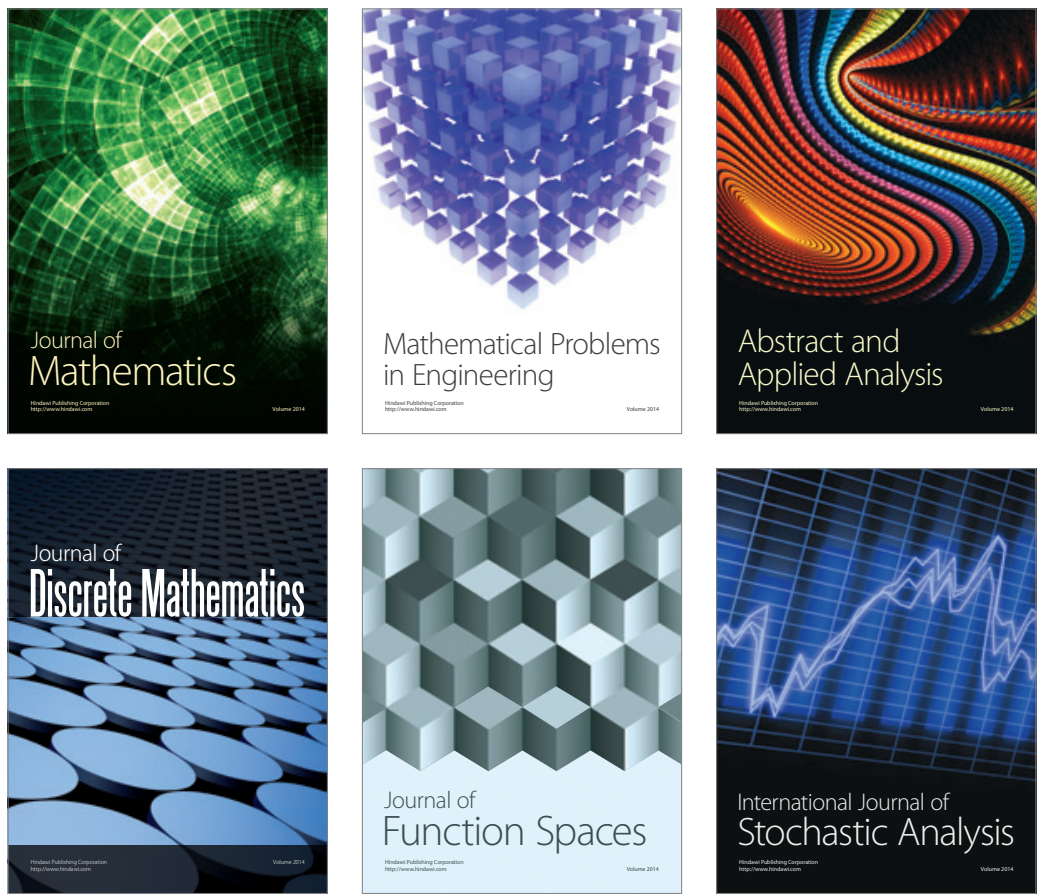

Journal of

Function Spaces

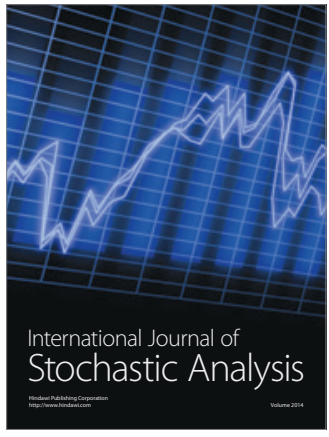

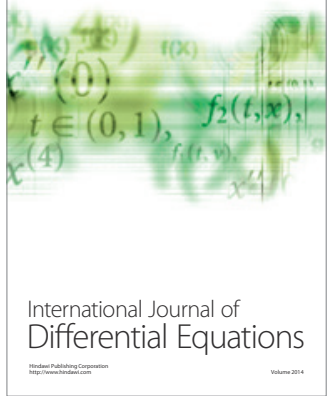
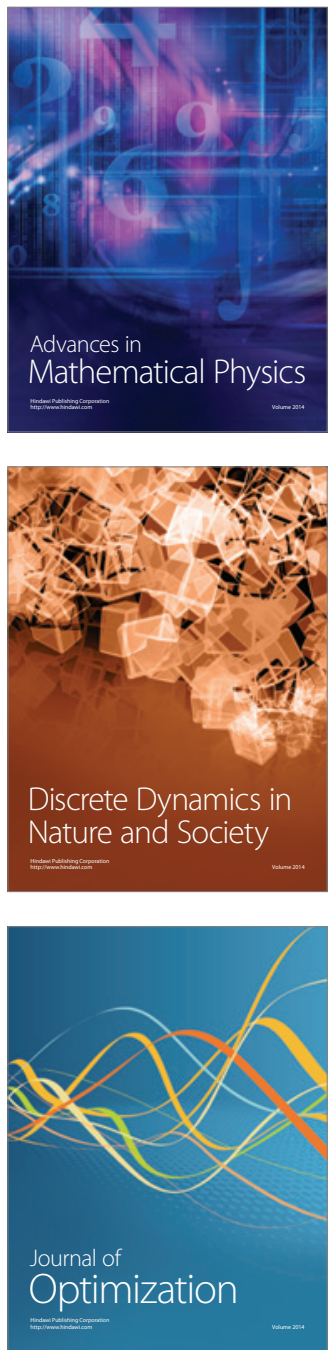